\title{
Aloe vera leaf extract mediated green synthesis of selenium nanoparticles and assessment of their In vitro antimicrobial activity against spoilage fungi and pathogenic bacteria strains
}

https://doi.org/10.1515/gps-2019-0007

Received August 09, 2018; accepted October 03, 2018.

\begin{abstract}
Selenium as an essential trace element for the health of the humans was used to hydrothermally synthesis of selenium nanoparticle (Se NPs) using Aloe vera leaf extract (ALE). Effects of synthesis parameters namely; amount of ALE (1-5 ml) and amount of $\mathrm{Na}_{2} \mathrm{SeO}_{3}$ solution $(10-30 \mathrm{ml})$, on the particle size and colour intensity of the solution containing Se NPs were studied using response surface methodology. FT-IR spectroscopy, UV-Vis spectrophotometry, DLS analyzer and TEM were used to determine the specifications of the ALE and synthesized Se NPs. Obtained results indicated that the ALE contained several bioactive compounds, which they had hydroxyl and amide I groups in their structures and these two functional groups had key roles in the reduction of the selenium ions to form Se NPs and stabilizing of them. Furthermore, spherical fabricated Se NPs using obtained optimum synthesis parameters, $4.92 \mathrm{~mL}$ of ALE and $13.03 \mathrm{~mL}$ of $\mathrm{Na}_{2} \mathrm{SeO}_{3}$ solution, had colour intensity, mean particle size, zeta potential and polydispersity index values of $3.0 \%$ a.u., $50 \mathrm{~nm},-18 \mathrm{mV}$ and 0.344 , respectively according to the DLS analysis. The synthesized Se NPs had also high antibacterial and antifungal activities against 4 selected pathogenic bacteria and spoilage fungi strains.
\end{abstract}

Keywords: selenium nanoparticles; hydrothermal synthesis; Aloe vera; antimicrobial activities

\footnotetext{
* Corresponding author: Hoda Jafarizadeh-Malmiri, Faculty of Chemical Engineering, Sahand University of Technology, East Azarbaijan, Tabriz, Iran. e-mail: h_jafarizadeh@sut.ac.ir; h_jafarizadeh@yahoo.com

Borna Fardsadegh, Faculty of Chemical Engineering, Sahand University of Technology, East Azarbaijan, Tabriz, Iran
}

\section{Introduction}

By developments and application of nanotechnology in the past decade, metal and non-metal nanoparticles (NPs) have gained more attention because of their different attributes as compared to those of them in the bulk form. However, these unique characteristics of the NPs are drastically depended on morphology, size and size distribution of the formed NPs [1]. Among different NPs, selenium nanoparticles (Se NPs) have gained a great deal of interests because of their specific biological, chemical, medicinal and pharmaceuticals properties [2]. Several studies have indicated that Se NPs can be strongly controlled the thyroid hormone metabolism, body's defense system and numerous cancer metastases $[3,4]$. In fact, selenium has a key role in the biosynthesis of the important selenoenzymes including glutathione peroxidase and thioredoxin reductase which, they are essential for the health of the humans and other mammals [5-7].

Several chemical, physical and green NPs synthesis methods have been developed and utilized to fabricate numerous NPs. However, green synthesis techniques are considered as one step, economic and eco-friendly method due to using natural bioreductants and stabilizers in the formation of the NPs [2,8]. Plants and their derivatives extracts have been widely used in the fabrication of the NPs as compared to the microorganisms, which it can be related to the elimination of the cell culturing and isolation [2]. Aloe vera, as an important medical plant, includes peel (leaf) and gel, which several studies have been revealed that its leaf contains numerous vitamins, polysaccharides, proteins, phenolic compounds, lignin, saponins, sterols, flavonoids, enzymes and organic acids which they have essential role in the reduction of the ions to their element and form their NPs, and stabilizing of the synthesized NPs [9-11]. In the synthesis of the NPs, the main goal is formation of the NPs with minimum particle size and maximum stability $[12,13]$. Several parameters are affected these characteristics of the fabricated NPs, including $\mathrm{pH}$, 
temperature, type and concentration of the leaf extract, type and concentration of the ions precursors and other operating parameters which they are strongly depended to the utilized methods [1,14,15]. Response surface methodology (RSM), as combination of the mathematics and statistics, is a useful technique to model and analyze of a dependent variable, which it is influenced by several independent parameters [16]. Furthermore, RSM has several benefits, which make it highlight as compared to the classic one parameter at-a-time optimization [12].

Therefore, the present study focused on i) assessment of the potential application of the Aloe vera leaf extract (ALE) in the fabrication of the Se NPs, ii) optimization of the synthesis parameters using RSM to obtain Se NPs, under subcritical water conditions, with lower particle size and the highest stability, and iii) evaluation of the physico-chemical, bactericidal and fungicidal activities of the made Se NPs against pathogenic bacteria isolated tomato and orange spoilage fungi strains.

\section{Materials and method}

Aloe vera green fresh leaves with same colour, shapes and out of the physical damage were purchased from a local market (Tabriz, Iran). Sodium selenite $\left(\mathrm{Na}_{2} \mathrm{SeO}_{3}\right)$, as selenium salt, was provided from Merck Company (Merck, Darmstadt, Germany). Nutrient agar (NA) and Potato dextrose agar (PDA) were provided from Himedia (Himedia Co., Mumbai, India) and Oxoid Ltd. (Hampshire, UK). To provide the solutions, deionized double distilled water (DI) was utilized.

\subsection{Aloe vera leaf extract preparation}

Presented contaminations on the surface of the Aloe vera leaves were removed by washing the leaves and after that, those were sliced into the small pieces and shade dried during a week, at room temperature. Using a domestic miller (MX-GX1521; Panasonic, Tokyo, Japan), 4.69 g Aloe vera dried powder was provided and added into $100 \mathrm{~mL}$ of boiling DI water for $5 \mathrm{~min}$ to obtain the leaf extract. Finally, the extract was filtered using Whatman No. 40 filter paper, and retained in the refrigerator $\left(4^{\circ} \mathrm{C}\right)$.

\subsection{Fabrication of the Se NPs using ALE}

Reported literature studies revealed that, $\mathrm{Na}_{2} \mathrm{SeO}_{3}$ solution $(10 \mathrm{mM})$ was prepared by addition of $0.263 \mathrm{~g}$ of its salt into the $100 \mathrm{~mL}$ of DI water $[2,4,17,18]$. In typical synthesis, different amounts (1-5 mL) of ALE were mixed with various amounts (10-30 mL) of $\mathrm{Na}_{2} \mathrm{SeO}_{3}$ solution. Finally, the reaction solution was put into the laboratory autoclave, adjusted at $121^{\circ} \mathrm{C}$ and 1.5 bar, for $15 \mathrm{~min}$.

\subsection{Physico-chemical assay}

\subsubsection{FT-IR analysis}

The major active biocompounds of the ALE were investigated and evaluated using Fourier transforminfrared spectroscopy (Shimadzu 8400S, Shimadzu Co., Kyoto, Japan) with $\mathrm{KBr}$ pellets in the range of $4000-400 \mathrm{~cm}^{-1}$.

\subsubsection{SPR of the synthesized Se NPs}

Fabrication of the Se NPs can be easily observe using UV-Vis spectrophotometry (Jenway UV-Vis spectrophotometer6705, Staffordshire, UK) because of the surface Plasmon resonance of the formed Se NPs [18].

\subsubsection{Colour intensity of the synthesized Se NPs}

Ultraviolet-visible (UV-Vis) spectroscopy can be utilized to monitor the changes in the colour of the solution containing NPs based on the absorbance, due to the formed NPs SPR $[18,19]$. In fact, the colour of the solution can be correlated to the concentration of the formed NPs which, that can be manifested to the absorption in the visible range [19,20]. Red colour of the reaction mixture solution indicates that the Se NPs by reduction of the Se ions and converting them to the elemental Se and formation of the Se NPs [21]. Several studies indicated that by synthesis of the Se NPs in the solution, its colour progressively varies from the dark yellow into the red [17,22]. The colour intensity of the colloidal solution containing synthesized Se NPs was measured using UV-Vis spectrophotometry adjusted at $420 \mathrm{~nm}$ (wavelength) and stated as absorbance unit (\% a.u.).

\subsubsection{DLS analysis of the synthesized Se NPs}

A dynamic light scattering (DLS) particle size analyzer (Malvern instruments, Zetasizer Nano ZS, Worcestershire, UK) utilized to obtain the exact values of the mean particle size (with covered stabilizing layer), zeta potential, 
polydispersity index (PDI) and the particle size distribution (PSD) for the fabricated Se NPs at $25^{\circ} \mathrm{C}$. PDI value changes from 0 to 1 and indicates the monodispersity (close to 0 ) and polydispersity (close to 1) of the formed NPs. Surface electric charge density of the fabricated NPs is known as zeta potential value and its high value indicates the higher physical stability of the formed NPs in the colloidal solution $[10,15,19,20]$.

\subsubsection{Morphology of the synthesized Se NPs}

In order to evaluate the shape and measure the exact value of the size (absolute particle size) for the fabricated Se NPs, transmission electron microscopy (TEM, CM120, Philips, Amsterdam, Netherlands) was utilized. For this reason, a drop of the solution containing fabricated Se NPs was put on a carbon-coated copper grid and monitored by the instrument which, its acceleration was $120 \mathrm{kV}$.

\subsection{Antibacterial assay}

Well diffusion method was utilized to evaluate the bactericidal effects of the fabricated Se NPs against to Gram positive (Staphylococcus aureus) and Gram negative (Escherchia coli) bacteria. For this analysis, $0.1 \mathrm{~mL}$ of the prepared bacterial suspension, containing $1.5 \times 10^{8} \mathrm{cfu}$ (colony forming unit), from the provided S. aureus (PTCC 1112) and E. coli (PPTCC 1270) which these strains obtained from the microbial Persian type culture collection (PTCC, Tehran, Iran), was inoculated on the surface of the plates containing NA. Three holes with $5 \mathrm{~mm}$ in diameter were made in the provided plates and $10 \mu \mathrm{L}$ of the ALE, $\mathrm{Na}_{2} \mathrm{SeO}_{3}$ solution and synthesized Se NPs solutions were decanted into the holes. The amended plates were put into the incubator, adjusted at $37^{\circ} \mathrm{C}$, for $24 \mathrm{~h}$. The created clear zone around the holes was then measured and indicated the antibacterial activities of the samples.

\subsection{Antifungal activity}

Fungicidal effects of the fabricated Se NPs was evaluated according to the inhibition in radial mycelial growth of Colletotrichum coccodes and Penicillium digitatum on the surface of the plates containing PDA incorporated with Se NPs. $C$. coccodes and $P$. digitatum were isolated from the local spoiled tomato and orange, respectively. According to the described method by Mohammadlou et al. [15]. From a pure culture of the mentioned fungi, $5 \mathrm{~mm}$ agar disks, in diameter, were isolated and put in the middle of the control (PDA) plates and those were poured with PDA and Se NPs and ALE. The provided plates were then incubated at room temperature $\left(26 \pm 2^{\circ} \mathrm{C}\right)$ for 7 days and their hyphae growth was measured daily. The fungicidal activities of the formed Se NPs were stated as fungal mycelia growth (mm) inhibition [23].

\subsection{Design of experiments and data analysis}

As compared to other statistical techniques, which those were based on classical one-variable-a-time, response surface methodology (RSM) has numerous advantages including generation of the various important data using minimum experiment runs and evaluation of the linear, quadratic and interaction effects of the independent parameters on the responses $[10,12,15,19]$. Therefore, central composite design (CCD), using a block, with RSM were chosen to design of experiments and evaluate the effects of the synthesis parameters namely; amount of $\operatorname{ALE}\left(\mathrm{X}_{1}, 1-5 \mathrm{~mL}\right)$ and amount of $10 \mathrm{mM} \mathrm{Na}_{2} \mathrm{SeO}_{3}$ solution $\left(\mathrm{X}_{2}, 10-30 \mathrm{~mL}\right)$, on the colour intensity $\left(\mathrm{Y}_{1}\right)$ and particle size $\left(\mathrm{Y}_{2}\right)$ of the mixture solutions containing Se NP. Response variables, colour intensity $\left(\mathrm{Y}_{1}\right)$ and mean particle size $\left(\mathrm{Y}_{2}\right)$ of the formed Se NPs, as function of the synthesized parameters, were followed a second-order polynomial equation (1), which in this equation, $B_{0}$ is a constant and $B_{i}, B_{i i}$ and $B_{i j}$ are the coefficients of the linear, quadratic and interaction terms.

$$
\mathrm{Y}=\mathrm{B}_{0}+\mathrm{B}_{1} \mathrm{X}_{1}+\mathrm{B}_{2} \mathrm{X}_{2}+\mathrm{B}_{1} \mathrm{X}_{1}^{2}+\mathrm{B}_{2} \mathrm{X}_{2}^{2}+\mathrm{B}_{2} \mathrm{X}_{1} \mathrm{X}_{2}
$$

Coefficient of determination $\left(R^{2}\right)$ and its adjusted $\left(R^{2}-\right.$ adj) were used to evaluate the suitability of the generated model. Analysis of variance based on the terms p-value $(p<0.05)$ and $F$ ratio was employed to significance determinations of the obtained models. Two-dimensional contour plots, based on the generated models, were established to well imagine the interaction effect of the synthesis parameters. Numerical multiple response and graphical optimizations were utilized to obtain exact values of the optimum synthesis parameters and optimum area, respectively. Three more approval experiments using obtained optimum synthesis parameters were done to verify accuracy of the generated models for prediction values of the responses within the defined ranges. Minitab v.16 statistical software (Minitab Inc., PA, USA) was used for the design of experiments, RSM, data analysis and optimization procedures. 


\section{Results and discussion}

\subsection{ALE specifications}

FT-IR spectra of the ALE, shows several functional groups which those can be act as reducing and stabilizing agents in the formation of the Se NPs (Figure 1). Figure 1 indicates three centered main peaks at 572.8, 1635.52, and $3454.3 \mathrm{~cm}^{-1}$ and five weak peaks 1041, 1251.7, 1402.2, 2065.6 and $2351.1 \mathrm{~cm}^{-1}$. The widest observed peak at wavenumbers of $2900-3600 \mathrm{~cm}^{-1}$ was related to the stretching vibrations of hydroxyl groups $(\mathrm{O}-\mathrm{H})$ which had key rule in the reduction of Se ions to their elements and formation of the Se NPs. Furthermore, a minor centered peak at $1402.15 \mathrm{~cm}^{-1}$ could be related to the existence of carboxylic components [15]. The centered peak at $1635.52 \mathrm{~cm}^{-1}$, was attributed to the amide I group, which was related to the proteins and has key role in the stabilizing of formed Se NPs [10,15,24]. The mean $\mathrm{pH}$ value of ALE was about 5, indicating that the ALE is an acidic solution.

\subsection{Generation of the models}

According to the experimental design and obtained data for the responses (Table 1) the models were generated. $\mathrm{R}^{2}$ and $\mathrm{R}^{2}$-adj for the fitted models are also given in Table 2. P-Value and $F$ ratio for the each term of the obtained models are presented in Table 3. As can be seen in Table 2, higher values of the $R^{2}$ and $R^{2}$-adj (>94.00) for the both generated models, verified their suitable accuracy. Furthermore, the insignificant lack of fits $(p>0.05)$ of the generated models revealed their appropriate fitness to the synthesis variables (Table 2). As can be seen in Table 3, all the main, quadratic and interaction effects of the amount of ALE solution and amount of $\mathrm{Na}_{2} \mathrm{SeO}_{3}$ solution were effective $(p<0.05)$ on the particle size of the fabricated Se NPs. However, quadratic term of the amount of $\mathrm{Na}_{2} \mathrm{SeO}_{3}$ solution and its interaction with amount of the ALE

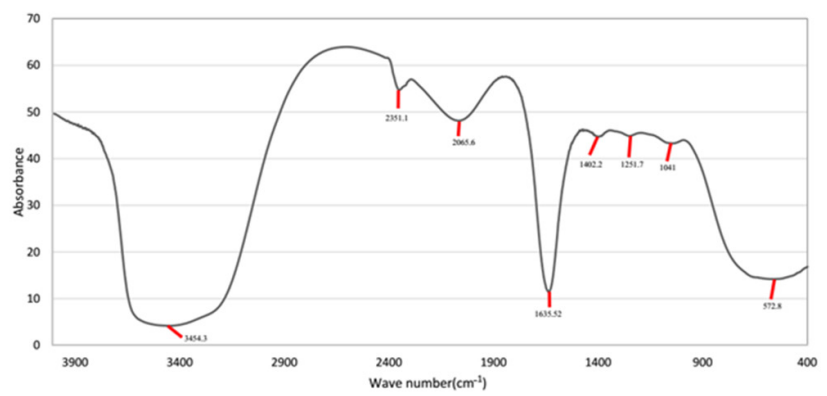

Figure 1: FT-IR spectrum of Aloe vera leaf extract.
Table 1: Central composite design (CCD) and response variables for processing of synthesis Se NPs.

\begin{tabular}{lcccc}
\hline $\begin{array}{l}\text { Sample } \\
\text { number }\end{array}$ & $\begin{array}{c}\text { Amount of } \\
\text { ALE }(\mathrm{mL})\end{array}$ & $\begin{array}{c}\mathrm{Amount}_{\mathrm{Na}} \mathrm{SeO}_{3}(\mathrm{~mL}) \\
\mathrm{Na}_{2}\end{array}$ & $\begin{array}{c}\text { Colour inten- } \\
\left.\text { sity (a.u. }{ }^{\mathrm{a}}\right)\end{array}$ & $\begin{array}{c}\text { Particle size } \\
(\mathrm{nm})\end{array}$ \\
\hline 1 & & & $\begin{array}{c}\text { Experimental } \\
\text { Experimental }\end{array}$ \\
2 & 3.00 & 20.00 & 2.129 & 498 \\
3 & 5.00 & 20.00 & 2.601 & 121 \\
4 & 1.59 & 27.07 & 0.718 & 893 \\
5 & 1.00 & 20.00 & 0.556 & 142 \\
6 & 1.59 & 12.92 & 1.760 & 1315 \\
7 & 3.00 & 20.00 & 2.163 & 831 \\
8 & 3.00 & 20.00 & 2.242 & 741 \\
9 & 3.00 & 20.00 & 2.064 & 742 \\
10 & 4.41 & 12.93 & 2.990 & 923 \\
11 & 3.00 & 10.00 & 2.890 & 3243 \\
12 & 3.00 & 30.00 & 1.635 & 911 \\
13 & 4.41 & 27.07 & 1.962 & 599 \\
\hline
\end{tabular}

${ }^{a}$ Absorbance unit

Table 2: Regression coefficients, $R^{2}, R^{2}$-adj, and probability values for the fitted models.

\begin{tabular}{lcc}
\hline Regression coefficient & Colour intensity (a.u. $\left.{ }^{\mathrm{a}}\right)$ & Particle size (nm) \\
\hline $\mathrm{B}_{0}$ (constant) & 1.00856 & 2073.27 \\
$\mathrm{~B}_{1}$ (main effect) & 1.3907 & 3424.18 \\
$\mathrm{~B}_{2}$ (main effect) & -0.09638 & -446.54 \\
$\mathrm{~B}_{11}$ (quadratic effect) & -0.1539 & -477.62 \\
$\mathrm{~B}_{22}$ (quadratic effect) & 0.00068 & 12.59 \\
Interaction effect $\beta_{12}$ & -.00035 & -56.88 \\
$\mathrm{R}^{2}$ & 99.31 & 97.40 \\
R-adj & 98.81 & 94.79 \\
Lack of fit (p-value) & 0.057 & 0.144 \\
\hline
\end{tabular}

${ }^{a}$ Absorbance unit

$B_{0}$ is constant and $B_{i}, B_{i j}$ and, $B_{i j}$ are the linear, quadratic and interaction coefficient of the quadratic polynomial equation respectively. 1. Amount of Aloe vera leaf extract $(\mathrm{mL}) ; 2$. Amount of $\mathrm{Na}_{2} \mathrm{SeO}_{3}(\mathrm{~mL})$

Table 3: $p$-Value and $F$ ratio of the regression coefficients in the obtained models.

\begin{tabular}{lccccc}
\hline \multirow{2}{*}{ Main effects } & \multicolumn{2}{c}{ Main effects } & \multicolumn{2}{c}{ Quadratic effects } & Interacted effects \\
\cline { 2 - 6 } & $\mathbf{X}_{1}$ & $\mathbf{X}_{2}$ & $\mathbf{X}_{11}$ & $\mathbf{X}_{22}$ & $\mathbf{X}_{12}$ \\
\hline $\begin{array}{l}\text { Colour intensity (a.u.) } \\
\text { p-value }\end{array}$ & & & & \\
F-ratio & 134.000 & 0.009 & 0.000 & 0.287 & 0.931 \\
& 12.97 & 107.65 & 1.33 & 0.01 \\
Particle size (nm) & & & & \\
p-value & 0.002 & 0.002 & 0.001 & 0.000 & 0.014 \\
F-ratio & 36.93 & 39.06 & 48.08 & 78.60 & 13.64 \\
\hline
\end{tabular}

1. Amount of Aloe vera leaf extract $(\mathrm{mL}) ; 2$. Amount of $\mathrm{Na}_{2} \mathrm{SeO}_{3}(\mathrm{~mL})$ 
solution had insignificant effects on the colour intensity of the mixture solution containing formed Se NPs.

\subsubsection{Colour intensity}

During Se NPs synthesis, the colour of solution containing fabricated Se NPs were changed from dark yellow (Figure 2a) into dark red (Figure 2b). The concentration of the synthesized Se NPs can be manifested in the colour intensity of their solutions. The effects of the synthesis variables on the colour intensity of the solutions are indicated in Figure 3. The obtained results revealed that, at constant amount of the $\mathrm{Na}_{2} \mathrm{SeO}_{3}$ solution, by increasing the amount of the ALE, colour intensity was increased. The obtained results were in line with findings of Ahmadi et al. [10]. They found that by increasing the amount of ALE, the concentration of bioreductants increased in the mixture

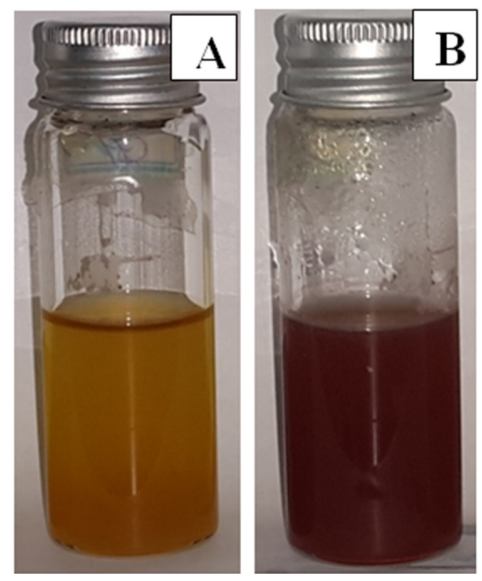

Figure 2: Illustration of colour change in mixed Aloe vera leaf extract and $\mathrm{Na}_{2} \mathrm{SeO}_{3}$ solution: (a) before synthesis, (b) after synthesis using autoclave.
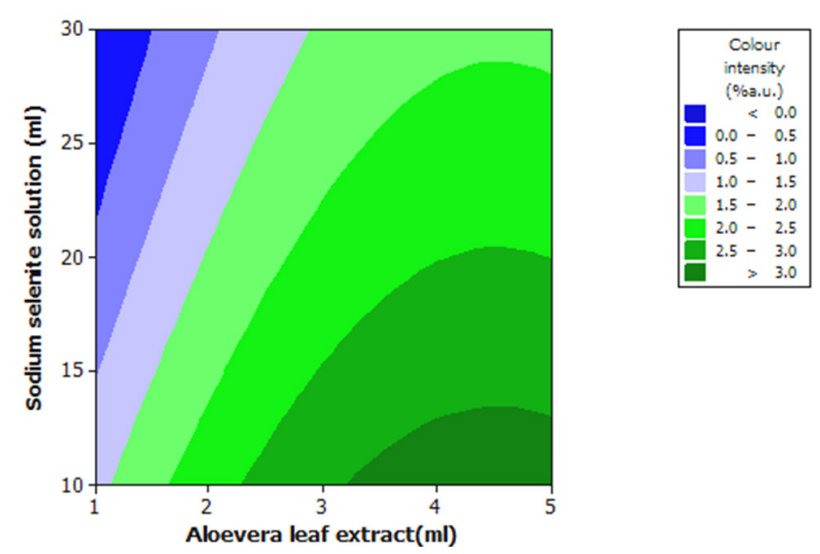

Figure 3: Contour plot of colour intensity of the solution containing Se NPs as function of amount of $\mathrm{Na}_{2} \mathrm{SeO}_{3}$ solution and Aloe vera leaf extract. solutions which in turn, increased the concentration of the formed NPs as can be observed by increasing in the colour intensity. On the other hand, at constant amounts of the ALE, by increasing the amount of $\mathrm{Na}_{2} \mathrm{SeO}_{3}$ solution, different patterns were observed and the colour intensity was decreased. The obtained result can be explained by the fact that by increasing the amount of selenium ions in the solutions, the repulsion of the system increased due to the positive charge of the ions and the rate of nucleation decreased $[12,15,19]$. As can be seen in Table 1 , the maximum colour intensity ( $2.99 \%$ a.u.) was obtained using $4.41 \mathrm{~mL}$ of ALE and $12.93 \mathrm{~mL}$ of $\mathrm{Na}_{2} \mathrm{SeO}_{3}$ solution.

\subsubsection{Particle size (nm)}

As can be seen in Table 1, the mean particle size values of the fabricated SeNPs ere varied from 121 to $3243 \mathrm{~nm}$. The effects of the amount of ALE and the amount of $\mathrm{Na}_{2} \mathrm{SeO}_{3}$ solution on the Se NPs particle size are shown in Figure 4. The obtained results demonstrated that, the minimum particle size was obtained at maximum amount of the ALE and $\mathrm{Na}_{2} \mathrm{SeO}_{3}$ solution. Table 1 also indicated that minimum particle size for the fabricated Se NPs $(121 \mathrm{~nm})$ was achieved using $5 \mathrm{ml}$ of ALE and $20 \mathrm{ml}$ of $\mathrm{Na}_{2} \mathrm{SeO}_{3}$ solution. The obtained result can be explained by the fact that by increasing the amount of ALE in the mixture solution, the nucleation rate was increased due to the high amount of bioreductants and on the other hand by increasing the amount of the $\mathrm{Na}_{2} \mathrm{SeO}_{3}$ solution, the repulsion of the mixture solution increased which it prevented the agglomeration of the formed Se NPs. The obtained results were in line with finding of Mohammadlou et al. [15] and Eskandari et al. [12] in the green synthesis of the silver NPs using Pelargonium leaves and gold NPs using mushroom extracts.
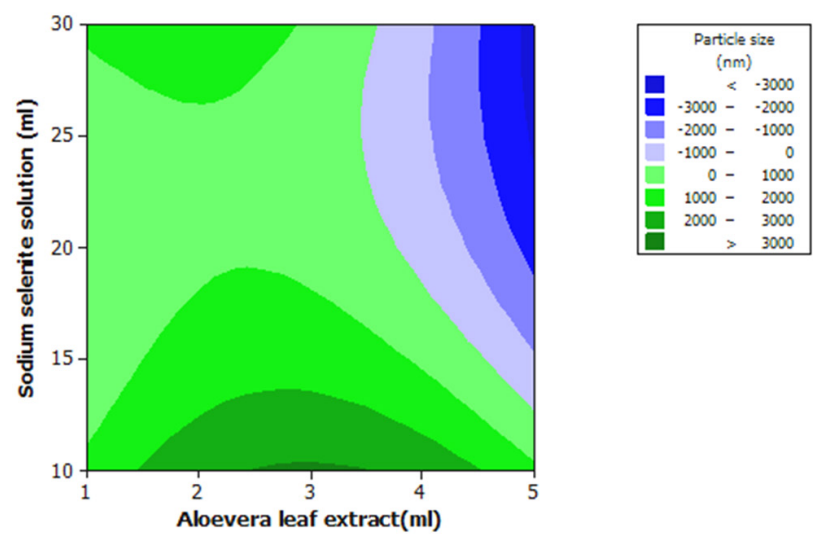

Figure 4: Contour plot of particle size of the solution containing Se NPs as function of amount of $\mathrm{Na}_{2} \mathrm{SeO}_{3}$ solution and Aloe vera leaf extract. 


\subsection{Optimization of processing parameters for the synthesized Se NPs}

The Se NPs synthesis parameters were considered as optimum conditions when the fabricated and achieved Se NPs highest colour intensity and the smallest mean particle size. Figure 5 indicates graphical optimization area based on an overlaid contour plot. The results revealed that the fabricated Se NPs with most desirable characteristics were obtained using synthesis conditions placed in the white coloured area. The obtained results by numerical multiple optimization also revealed that the fabrication conditions using a $4.92 \mathrm{~mL}$ of ALE and $13.03 \mathrm{~mL}$ of $\mathrm{Na}_{2} \mathrm{SeO}_{3}$ solution for the formation of $\mathrm{Se}$ NPs would give the most desirable Se NPs with colour intensity and mean particle size of the $3.0 \%$ a.u. and $33.30 \mathrm{~nm}$ (according to TEM analysis), respectively. Moreover, verification tests using three synthesized Se NPs solutions at obtained optimum synthesis conditions revealed that there were insignificant differences between the predicted and the experimental values of the responses which in turn, confirmed the adequacy of the generated models.

\subsection{Qualifications of the fabricated Se NPs at obtained optimum conditions}

\subsubsection{UV-Vis spectrophotometry analysis}

Formation of Se NPs using ALE and $\mathrm{Na}_{2} \mathrm{SeO}_{3}$ solution could be evaluated based on the fabricated Se NPs SPR, using UV-Vis spectral analysis. As can be seen in Figure 6, the broad emission peaks $\left(\lambda_{\max }\right)$ of the synthesized Se NPs
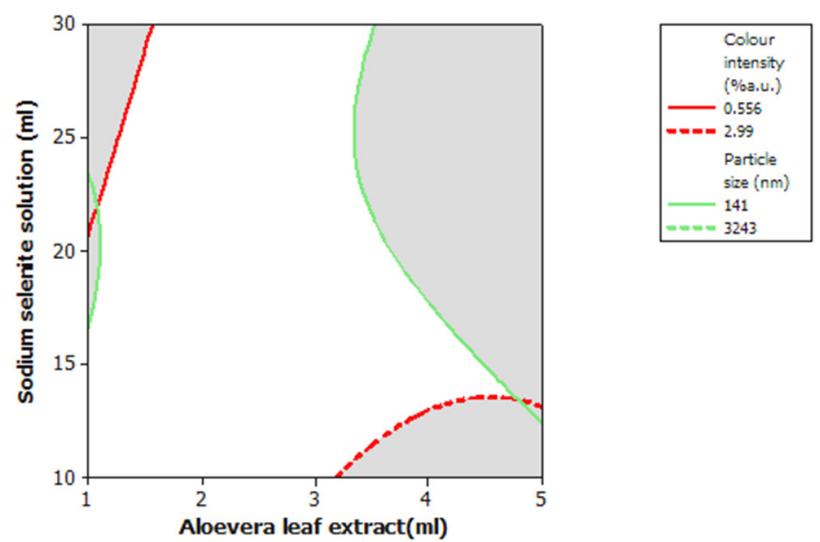

Figure 5: Overlaid contour plot of colour intensity, particle size with acceptable levels as function of amount of $\mathrm{Na}_{2} \mathrm{SeO}_{3}$ solution and Aloe vera leaf extract. was achieved at $323 \mathrm{~nm}$ which was centered between 295 to $340 \mathrm{~nm}$ [25-28].

\subsubsection{DLS analysis}

At obtained optimum synthesis conditions, the zeta potential value of the fabricated Se NPs was $-18 \mathrm{mV}$ which indicated that the formed NPs were surrounded with negatively charged groups and had high stability. The PDI value of the fabricated Se NPs was 0.344. The small value of the PDI indicated that the formed Se NPs were monodispersed. The size distribution of the synthesized Se NPs is also shown in Figure 7. However, our previous study on green synthesis of Se NPs using Pelargonium zonale indicated that Se NPs with mean particle size, PDI and zeta potential values of $136 \mathrm{~nm}, 0.321$ and $-24.6 \mathrm{mV}$, respectively were fabricated at obtained optimum synthesis conditions using $1.48 \mathrm{~mL}$ Pelargonium zonale leaf extract and $15 \mathrm{~mL}$ of $\mathrm{Na}_{2} \mathrm{SeO}_{3}$ solution [29]. The obtained results indicated that Aloe vera leaf extract could be effectively synthesized Se NPs with smaller particle size and higher stability, due to high value of the zeta potential, as compared to the Pelargonium zonale leaf extract. It could be related to strong reductant and stabilizing agents existed in Aloe vera leaf extract.

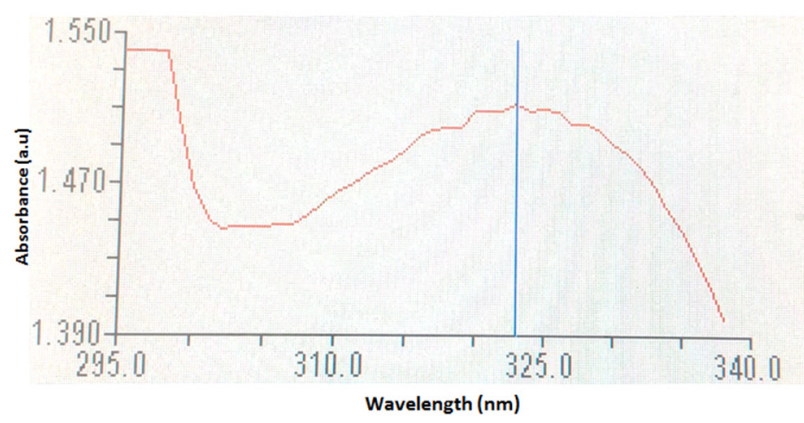

Figure 6: UV-Vis spectra of the mixture solution including $\mathrm{Na}_{2} \mathrm{SeO}_{3}$ and Aloe vera leaf extraxt, after synthesis with exposure to microwave irradiation at obtained optimum synthesis conditions.

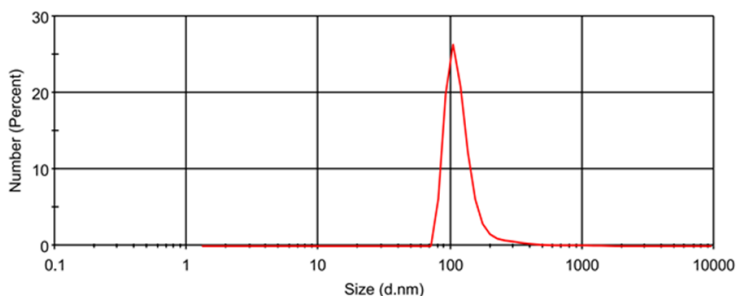

Figure 7: TEM image of the synthesized Se NPs at obtained optimum conditions. 


\subsubsection{Morphology}

TEM analysis was accomplished to identify the morphology of the formed Se NPs. TEM image of the fabricated Se NPs at obtained synthesis conditions showed that the particles were monodispersed and spherical with mean particle size of $50 \mathrm{~nm}$ in diameter (Figure 8). This spherical shape demonstrated that the thermodynamic stable fabricated Se NPs had minimum surface energy, which was in line with high value of the zeta potential [25-28].

\subsubsection{Antibacterial activity}

Bactericidal activities of the fabricated Se NPs against to the $S$. aureus and E. coli during incubation are presented in Figure 9. The results revealed that, the inhibition zones

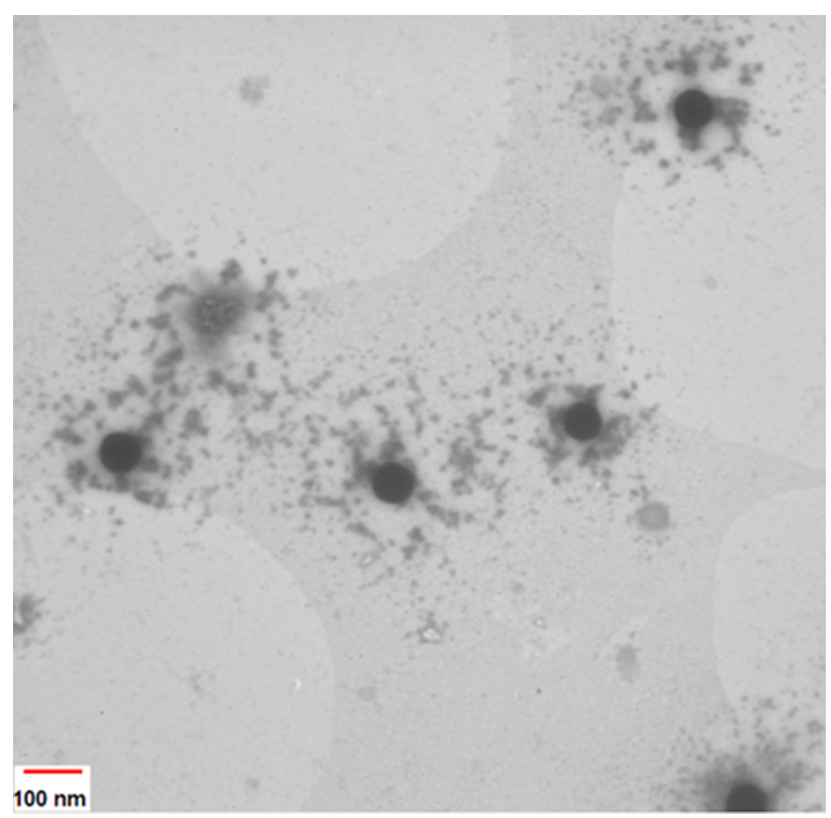

Figure 8: Size distribution of the synthesized Se NPs.

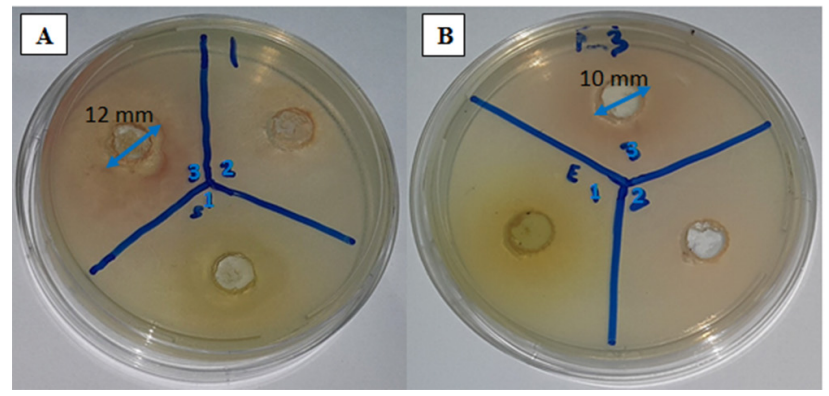

Figure 9: Created zones of inhibition with S. aureus (A) and E. coli (B) incubated at $37^{\circ} \mathrm{C}$ for $24 \mathrm{~h}$. could be observed for both $S$. aureus and $E$. coli bacteria. However, the diameter of the inhibition zones around the S. aureus $(12 \mathrm{~mm})$ was higher than that of around E. coli $(10 \mathrm{~mm})$. The obtained results werealso in line with findings of Khiralla et al. [30] and, Srivastava and Mukhopadhyay [25], they found that the green synthesized Se NPs had high bactericidal effects on the both mentioned Gramnegative and Gram-positive bacteria strains.

\subsubsection{Antifungal activity}

Fungicidal activities of the fabricated Se NPs on mycelia growth of $P$. digitatum and $C$. coccodes during incubation are presented in Figures 10a and 10b, respectively. As can be seen in Figure 10a, Se NPs amended in PDA media could inhibit the mycelia growth of $P$. digitatum, while, the synthesized Se NPs showed low fungicidal effect on $C$. coccodes (Figure 10b). Several studies demonstrated that the mechanism of the fungicidal effects of Se ions were included of absorption and accumulation of them by the
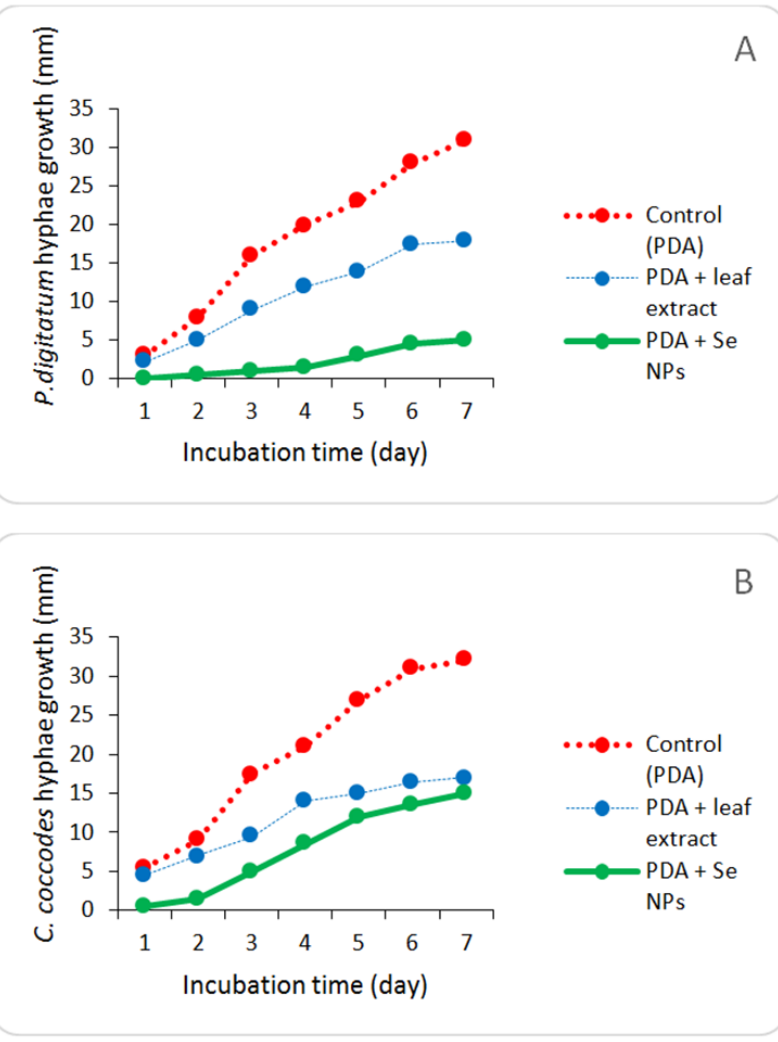

Figure 10: Mycelia growth inhibition of the synthesized Se NPs toward Penicillium digitatum (a) and Colletotrichum coccodes (b) after 7 days of incubation at $26 \pm 2{ }^{\circ} \mathrm{C}$. Data are the mean value of three replicates (each replicate contains four plates). 
fungus cell which in turn, lead shrinking of the cytoplasm membrane and inhibited the cell vital activities [15,20].

\section{Conclusions}

The obtained results from the present study revealed that Aloe vera leaf extract had several natural bioactive compounds which those could be effectively utilized in the fabrication of Se NPs without using other chemical reducing agents. Hydrothermal synthesis of Se NPs by autoclave is one of the fast and clean practical synthesis methods due to its capability to generate simultaneously high pressure and high temperature. In addition to, RSM could effectively predict the effect of the synthesis variables on the particle size and colour intensity of the colloid solutions containing Se NPs. RSM was successfully applied to develop empirical models for the prediction of the Se NPs synthesis conditions. Finally, the developed synthesized method can be utilized in the fabrication of other metal and metal oxide NPs.

Acknowledgements: The authors appreciate the financial support of the Iran Nanotechnology Initiatives Council (grant no. 125018).

\section{References}

[1] Chen H., Yoo J.B., Liu Y., Zhao G., Green synthesis and characterization of Se nanoparticles and nanorods. Electron Mater. Lett., 2011, 7, 333-336.

[2] Prasad K.S., Patel H., Patel T., Patel K., Selvaraj K., Biosynthesis of Se nanoparticles and its effect on UV-induced DNA damage. Colloids Surf. B Biointerfaces, 2013, 103, 261-266.

[3] Benstoem C., Goetzenich A., Kraemer S., Borosch S., Manzanares W., Hardy G., et al., Selenium and its supplementation in cardiovascular disease-What do we know? Nutrients, 2015, 7, 3094-3118.

[4] Zhou Y., Xu M., Liu Y., Bai Y., Deng Y., Liu J., et al., Green synthesis of Se/Ru alloy nanoparticles using gallic acid and evaluation of theiranti-invasive effects in HeLa cells. Colloids Surf. B Biointerfaces, 2016, 144, 118-124.

[5] Falandysz J., Selenium in edible mushrooms. J. Environ. Sci. Health, part C: Environ. Carcinog. Ecotoxicol. Rev., 2008, 26, 256-299.

[6] Fayet-Moore F., Petocz P., Samman S., Micronutrient status in female university students: Iron, zinc, copper, selenium, vitamin b(12) and folate. Nutrients, 2014, 6, 5103-5116.
[7] Chen W., Li Y., Yang S., Yue L., Jiang Q., Xia W., Synthesis and antioxidant properties of chitosan and carboxymethyl chitosanstabilized selenium nanoparticles. Carbohydr. Polym., 2015, 132, 574-581.

[8] Sharma G., Sharma A.R., Bhavesh R., Park J., Ganbold B., Nam J.S., et al., Biomolecule-mediated synthesis of selenium nanoparticles using dried Vitis vinifera (raisin) extract. Molecules, 2014, 19, 2761-2770.

[9] Sánchez-Machado D.I., López-Cervantes J., Sendón R., SanchesSilva A., Aloe vera: Ancient knowledge with new frontiers. Trends Food Sci. Technol., 2017, 61, 94-102.

[10] Ahmadi O., Jafarizadeh-Malmiri H., Jodeiri N., Eco-friendly microwave-enhanced green synthesis of silver nanoparticles using Aloe vera leaf extract and their physico-chemical and antibacterial studies. Green Process. Synth., 2018, 7, 231-140.

[11] Badole S.L., Bagul P.P., Menaa F., Aloe vera: Use for skin disease. In: Watson R.R., Zibadi S. (Eds.), Bioactive dietary factors and plant extracts in dermatology. Humana Press, Totowa, N.J., 2013.

[12] Eskandari-Nojehdehi M., Jafarizadeh-Malmiri H., RahbarShahrouzi J., Optimization of processing parameters in green synthesis of gold nanoparticles using microwave and edible mushroom (Agaricus bisporus) extract and evaluation of their antibacterial activity. Nanotechnol. Rev., 2016, 5, 537-548.

[13] McClements J., McClements D.J., Standardization of nanoparticle characterization: methods for testing properties, stability, and functionality of edible nanoparticles. Crit. Rev. Food Sci. Nutr., 2016, 56, 1334-1362.

[14] Fatimah I., Green synthesis of silver nanoparticles using extract of Parkia speciosa Hassk pods assisted by microwave irradiation. J. Adv. Res., 2016, 7, 961-969.

[15] Mohammadlou M., Jafarizadeh-Malmiri H., Maghsoudi H., Hydrothermal green synthesis of silver nanoparticles using Pelargonium/Geranium leaf extract and evaluation of their antifungal activity. Green Process. Synth., 2017, 6, 31-42.

[16] Jafari N., Jafarizadeh-Malmiri H., Hamzeh-mivehroud M., Adibpour M., Optimization of UV irradiation mutation conditions for cellulase production by mutant fungal strains of Aspergillus niger through solid state fermentation. Green Process. Synth., 2017, 6, 334-340.

[17] Anu K., Singaravelu G., Murugan K., Benelli G., Green-synthesis of selenium nanoparticles using garlic cloves (Allium sativum): Biophysical characterization and cytotoxicity on vero cells. J. Cluster. Sci., 2017, 28, 551-563.

[18] Prasad K.S., Selvaraj K., Biogenic synthesis of selenium nanoparticles and their effect on As(III)-induced toxicity on human lymphocytes. Biol. Trace Elem. Res., 2014, 157, 275-283.

[19] Eskandari-Nojehdehi M., Jafarizadeh-Malmiri H., Jafarizad A., Microwave accelerated green synthesis of gold nanoparticles using gum arabic and their physico-chemical properties assessments. Z. Phys. Chem., 2018, 232, 325-343. 
[20] Eskandari-Nojedehi M., Jafarizadeh-Malmiri H., RahbarShahrouzi J., Hydrothermal green synthesis of gold nanoparticles using mushroom (Agaricus bisporus) extract: physico-chemical characteristics and antifungal activity studies. Green Process. Synth., 2018, 7, 38.

[21] El-Ramady H., Abdalla N., Taha H.S., Alshaal T., El-Henawy A., Faizy SE-D.A., et al., Selenium and nano-selenium in plant nutrition. Environ. Chem. Lett., 2016, 14, 123-147.

[22] Kriti Soni A.K.K., Green synthesis of selenium nanoparticles from Broccoli, characterization, application and toxicity. Adv. Tech. Biol. Med., 2017, 5, 198.

[23] Edirisinghe M., Ali A., Maqbool M., Alderson P.G., Chitosan controls postharvest anthracnose in bell pepper by activating defense-related enzymes. J. Food Sci. Technol., 2014, 51, 40784083.

[24] Jafarizad A., Safaee K., Gharibian S., Omidi Y., Ekinci D., Biosynthesis and in-vitro study of gold nanoparticles using Mentha and Pelargonium extracts. Procedia Mater. Sci., 2015, 11, 224-230.

[25] Srivastava N., Mukhopadhyay M., Green synthesis and structural characterization of selenium nanoparticles and assessment of their antimicrobial property. Bioprocess Biosyst. Eng., 2015, 38, 1723-1730.

[26] Shoeibi S., Mashreghi M., Biosynthesis of selenium nanoparticles using Enterococcus faecalis and evaluation of their antibacterial activities. J. Trace Elem. Med. Biol., 2017, 39, 135-139.

[27] Tran P.A., Webster T.J., Selenium nanoparticles inhibit Staphylococcus aureus growth. Int. J. Nanomed., 2011, 6, 15531558.

[28] Mittal A.K., Kumar S., Banerjee U.C., Quercetin and gallic acid mediated synthesis of bimetallic (silver and selenium) nanoparticles and their antitumor and antimicrobial potential. J. Colloid. Interface Sci., 2014, 431, 194-199.

[29] Fardsadegh B., Vaghari H., Mohammad-Jafari R., Najian Y., Jafarizadeh-Malmiri H., Biosynthesis, characterization and antimicrobial activities assessment of fabricated selenium nanoparticles using Pelargonium zonale leaf extract. Green Process. Synth. (in press), DOI:10.1515/gps-2018-0060.

[30] Khiralla G.M., El-Deeb B.A., Antimicrobial and antibiofilm effects of selenium nanoparticles on some foodborne pathogens. LWTFood Sci. Technol., 2015, 63, 1001-1007. 